\title{
Atención, memoria y funciones ejecutivas en estudiantes en prueba académica de Ingeniería Ambiental y Mecatrónica
}

Attention, memory, and executive functions of students in an academic study of environmental engineering and mechatronics programs

\section{Atenção, memória e funções executivas em estudantes em período de prova de engenharia ambiental e mecatrônica}

Nubia Yanneth Álvarez Vargas*, Diana Camila Rodríguez Díaz, \& Maritza Margarita Valero Otálora

Citar este artículo así:

Álvarez, N. Y., Rodríguez, D. C., y Valero, M. M. (2017). Atención, memoria y funciones ejecutivas en estudiantes en prueba académica de Ingeniería Ambiental y Mecatrónica. Revista Enfoques, 3(1), 159-172. http://dx.doi.org/

Derechos de autor: Licencia Creative Commons AtribuciónNoComercial-SinDerivadas 4.0 internacional y 2.5 Colombia (CC BYNC-ND 2.5 (O)

Recibido: agosto 29 de 2017 Revisado: septiembre 01 de 2017 Aceptado: septiembre 08 de 2017

* Contacto: Nubia Álvarez es Docente de la Facultad de Ciencias Humanas y Educativas de la Universidad de Boyacá. email: nyalvarez@uniboyaca.edu.co 
Resumen | Esta investigación describe los procesos cognitivos de atención y memoria, así como las funciones ejecutivas en estudiantes en prueba académica, que cursan los programas de Ingeniería Ambiental e Ingeniería Mecatrónica de la Universidad de Boyacá. Metodológicamente es de tipo cuantitativo, con un corte descriptivo. La muestra está constituida por 23 universitarios con bajo rendimiento académico, escogidos a través de un muestreo estratificado con afijación proporcional, con edad promedio entre18 y 25 años, con una ubicación semestral de primero a octavo semestre, de los cuales el $74 \%$ fueron hombres y un $26 \%$ mujeres. Los resultados encontrados permiten establecer que: en el proceso atencional, el $60 \%$ de los estudiantes de Ingeniería Ambiental (IA) y el 75\% de los estudiantes del programa de Ingeniería Mecatrónica (IM) presentan niveles altos en atención sostenida, pero para el caso de atención selectiva un $47 \%$ (IA) y un $12 \%$ (IM) de los estudiantes tienen un desempeño bajo; en memoria, se registró que el 93\% (IA) al parecer tiene dificultades para recordar detalles de los eventos y conceptos; en funciones ejecutivas, el 33\% (IA) y un $25 \%$ (IM) presentaron bajos niveles en la monitorización, manipulación y actualización de información.

Palabras Clave | atención, memoria, funciones ejecutivas.

Abstract | This research aims to describe the cognitive processes of: attention, memory and executive functions in students through academic tests of the Environmental Engineering and Mechatronics Engineering programs of Boyacá University. Methodologically, this is of the quantitative type, with a descriptive edge. The sample is made up of 23 university students from the Environmental Engineering and Mechatronics Engineering programs with low academic performance, chosen through a stratified sample with proportional allocation. The average age is 18 to 25 years old, all tested during their eighth semester, and of which $74 \%$ were men and $26 \%$ women. With respect to attention, it was found that $60 \%$ of Environmental Engineering students (IA) and $75 \%$ of Mechatronics Engineering (IM) students had high levels of sustained attention, but in the case of selective attention, 47\% (AI) and 12\% (IM) showed low performance; (IA) and 25\% (IM) respectively presented low levels in monitoring, manipulation and updating information.

Keywords | attention, memory, executive functions 
Resumo | O baixo rendimento acadêmico é uma problemática mundial em decorrência da multiplicidade de fatores sociais, econômicos e individuais, que devido a sua complexidade virou tema de interesse. É por isso, que a presente pesquisa tem como objetivo descrever os processos cognitivos de atenção, memoria e funções executivas de alunos em período de prova dos Programas de Engenharia Ambiental e Engenharia Mecatrônica da Universidade de Boyacá. A metodologia da pesquisa tem um enfoque quantitativo de tipo descritivo. A amostra esteve constituída por 23 alunos dos programas de Engenharia Ambiental e Engenharia Mecatrônica com baixo rendimento acadêmico, escolhidos a partir de uma seleção estratificada proporcional, com idades entre 18 e 25 anos, de primeiro ao oitavo semestre acadêmico, dos quais $74 \%$ foram homens e $26 \%$ mulheres. Os resultados indicam que nos processos de atenção um 60\% dos alunos de Engenharia Ambiental (IA) e $75 \%$ dos alunos do programa de Engenharia Mecatrônica (IM) apresentam níveis altos de atenção sustentada, mas no caso da atenção seletiva um $47 \%$ (IA) e um $12 \%$ (IM) dos alunos têm um desempenho baixo. No caso da memória se encontrou que um 93\% (IA) têm dificuldades para lembrar detalhes dos eventos e dos conceitos. Nas funções executivas um 33\% (IA) e um $25 \%$ (IM) respectivamente apresentaram níveis baixos na monitorização, manipulação e atualização da informação.

Palavras chave | atenção, memoria, funções executivas

\section{Introducción}

Uno de los temas que se ha venido fortaleciendo con el paso del tiempo y que ha permitido que la Psicología evolucione en la comprensión del rendimiento académico, respecto a la adquisición del aprendizaje, ha sido los procesos cognitivos de atención, memoria y funciones ejecutivas (Vallejos, 2012); lo que a su vez ha generado el interés de los entes gubernamentales por establecer diversas estrategias que permitan mejorar el rendimiento académico. Es por ello que se requiere una exploración profunda de dichos procesos.

La revisión de investigaciones afines con los estudios de los procesos cognitivos evidencia la relación entre estos procesos con otras variables ambientales y contextuales del estudiante, demostrando que es necesario seguir profundizando en este tipo de temáticas (Fernández y Gutiérrez, 2009; Furlan, Kohan, Cortada, Piemontesi y Heredia, 2008; Gavotto y Castellanos, 2015).

Es por las razones anteriormente expuestas que la presente investigación busca describir los procesos cognitivos de atención, memoria y funciones ejecutivas en estudiantes de los 
programas de IA e IM de la Universidad de Boyacá, que se encuentran en prueba académica por su bajo rendimiento académico. Esta investigación se realiza con el fin de conocer aspectos relacionados con la cognición y, de esta manera, contribuir con la comprensión y evaluación de esta temática, generando así resultados útiles para la ejecución de programas de intervención.

\section{Método}

\section{Tipo de investigación y diseño}

La investigación es de corte cuantitativo, de tipo descriptivo, diseñada con base en los parámetros establecidos por Hernández, Fernández y Baptista (2014) para la construcción de este tipo de investigaciones que deben desarrollarse bajo un orden riguroso.

\section{Participantes}

La población objeto de estudio está compuesta por 258 estudiantes universitarios de la Universidad de Boyacá, que se encuentran en prueba académica. De entre ellos, 85 pertenecen a la Facultad de Ingeniería. Luego de realizar un muestreo estratificado de afijación proporcional, se seleccionaron 23 individuos de IA e IM que cumplían con los criterios de inclusión, demarcados para la investigación.

\section{Instrumentos}

Para la medición de las variables del estudio se utilizaron los siguientes instrumentos: Ficha de Datos Sociodemográficos (FDS), Test de Clasificación de Tarjetas de Wisconsin (WCST), Test de Fluidez verbal (FAS), Test de los Senderos Tesen (TST), Test de los Cinco Dígitos (TCD), Prueba de Figuras incompletas (WAIS III), Prueba de Dígitos y Símbolo-Clave (WAIS III), y el Test de Aprendizaje Verbal de California (TAVC).

\section{Plan de análisis de resultados}

El análisis e interpretación de los resultados, obtenidos tras la aplicación de los instrumentos mencionados, se logró mediante la utilización de softwares estadísticos como Microsoft Office Excel y Statistical Package for the Social Sciences (SPSS). 


\section{Resultados y Discusión}

El registro de condiciones sociodemográficos es una herramienta que permite proporcionar información concreta de la población (Morales, Alviter, Hidalgo, García, y Molinar, 2013). En la tabla 1 se presentan las características sociodemográficas de los participantes.

Tabla 1 |Resultados generales - Datos sociodemográficos

\begin{tabular}{cl}
\hline RESULTADOS & \\
\hline Muestra & 23 estudiantes \\
Ubicación Semestral & Primero-octavo \\
Rango de Edad & $18-25$ \\
\hline
\end{tabular}

Nota: Resultados de datos sociodemográficos que incluyen muestra, ubicación semestral y rango de edad.

Los resultados obtenidos de este estudio permitieron establecer que el $74 \%$ de los sujetos fueron hombres y el $26 \%$ mujeres. Así mismo, se encontró que el rango de edad de los estudiantes con bajos promedios académicos está entre los 18 y 25 años, y su ubicación semestral está de primero a octavo semestre (Tabla 1).

De otra parte, se pudo evidenciar que la mayoría de los estudiantes que se encuentran en prueba académica pertenecen a las ingenierías (Tabla 2). Esto se relaciona con lo referido por Sáez, Guísasela y Garmendia (2013), quienes mencionan que los estudiantes de programas de Ingeniería presentan habilidad insuficiente para detectar y analizar problemas complejos, así como para transferir lo aprendido a diferentes contextos. Por ende, no sorprende encontrar un menor desempeño académico.

Tabla 2 | Datos sociodemográficos por programa

\begin{tabular}{lcc}
\hline Muestra por programa & & edad (años) \\
\hline Ing.Ambiental & 15 & $18-25$ \\
\hline Ing.Mecatronica & 8 & $19-22$ \\
\hline
\end{tabular}

Nota: Resultados de datos sociodemográficos por programa de Ingeniería Ambiental e Ingeniería Mecatrónica, especificando la edad y la muestra. 
En cuanto a las condiciones de bajo rendimiento académico, catalogado como prueba académica en la Universidad de Boyacá (Tabla 3), se encontró que el $43 \%$ de estudiantes en prueba académica pierden dos o más veces una asignatura, lo cual puede ser explicado por Castillo y Bracamonte (2011), quienes afirman que la complejidad matemática a la cual se enfrentan los estudiantes de Ingeniería puede afectar su rendimiento académico.

Tabla 3 | Tipos de prueba académica

\begin{tabular}{lc}
\hline Tipo de prueba académica & Porcentaje \\
\hline Segunda vez por bajo promedio acumulado & $4 \%$ \\
\hline Tercera vez por bajo promedio acumulado & $0 \%$ \\
\hline Segunda vez por perder una asignatura por dos o más veces & $43 \%$ \\
\hline Tercera vez por perder una asignatura por dos o más veces & $17 \%$ \\
\hline $\begin{array}{l}\text { Segunda vez por bajo promedio acumulado y perder una asignatura por } \\
\text { más de dos veces }\end{array}$ & $30 \%$ \\
\hline $\begin{array}{l}\text { Tercera vez por bajo promedio acumulado y perder una asignatura por más } \\
\text { de dos veces }\end{array}$ & $0 \%$ \\
\hline
\end{tabular}

Con respecto al proceso cognitivo de atención, se inicia el análisis con la atención sostenida (Tabla 4) donde el $75 \%$ de los estudiantes de IM y un $60 \%$ de IA presentan alta capacidad para mantener una respuesta de forma consistente durante un período de tiempo prolongado. Sin embargo, el 7\% de IA está dentro de los niveles bajos, lo que probablemente interfiere con el desarrollo de tareas cognitivas complejas relacionadas con el análisis y la síntesis de información, dando como resultado el no responder de forma adecuada.

Tabla 4|Atención sostenida

\begin{tabular}{ccc}
\hline Programa & \multicolumn{2}{c}{ Porcentaje } \\
\hline \multirow{2}{*}{ Ingeniería Ambiental } & Bajo & $7 \%$ \\
\cline { 2 - 3 } & Medio & $33 \%$ \\
\cline { 2 - 3 } & Alto & $60 \%$ \\
\hline
\end{tabular}




\begin{tabular}{ccc}
\hline Programa & \multicolumn{2}{c}{ Porcentaje } \\
\hline \multirow{2}{*}{ Ingeniería Mecatrónica } & Bajo & $0 \%$ \\
\cline { 2 - 3 } & Medio & $25 \%$ \\
\cline { 2 - 3 } & Alto & $75 \%$ \\
\hline
\end{tabular}

En relación con el proceso cognitivo de atención de tipo alternante (Tabla 5), se estableció que el $67 \%$ de IA y un $62 \%$ de los estudiantes de IM tienen un promedio medio en su desempeño, es decir, que presentan facilidad para cambiar el foco de atención entre tareas que implican requerimientos cognitivos diferentes, controlando la información procesada en cada momento. Sin embargo, entre el $20 \%$ y el $25 \%$ de los estudiantes de dichos programas presentaron un nivel bajo, lo que hace que no puedan cambiar rápidamente y de forma fluida los procesos entre tareas. Es así como se les dificulta extraer los elementos esenciales para el desarrollo de una actividad, o acción, que requiera vigilancia de aspectos que exigen un proceso preciso y organizado para cumplir su finalidad con eficiencia (Cuervo y Quijano, 2008).

Tabla 5|Atención alternante

\begin{tabular}{ccc}
\hline Programa & \multicolumn{2}{c}{ Porcentaje } \\
\hline \multirow{2}{*}{ Ingeniería Ambiental } & Bajo & $20 \%$ \\
\cline { 2 - 3 } & Medio & $67 \%$ \\
\cline { 2 - 3 } & Alto & $13 \%$ \\
\hline \multirow{2}{*}{ Ingeniería Mecatrónica } & Bajo & $25 \%$ \\
\cline { 2 - 3 } & Medio & $62 \%$ \\
\hline
\end{tabular}

Referente a la atención dividida (Tabla 6), se encontraron resultados dentro de los niveles medios, es decir, un $80 \%$ y $87 \%$ de estudiantes de IA e IM, respectivamente. Por tanto, se puede inferir que los estudiantes pueden atender a dos cosas al mismo tiempo y llevar a cabo actividades relacionadas con la selección de información, cuando se ofrecen varias opciones a la vez. Sin embargo, el $13 \%$ de los estudiantes presentó dificultades. Lo que sugiere problemas 
en las tareas que requieren el manejo de diferentes elementos, haciendo que los resultados de dichas tareas no sean los esperados. Es necesario mencionar que otro de los factores que pueden generar estos resultados es el uso inadecuado de estrategias de procesamiento de la información y autorregulación (Vásquez, 2009).

Tabla 6 |Atención dividida

\begin{tabular}{ccc}
\hline Programa & \multicolumn{2}{c}{ Porcentaje } \\
\hline \multirow{2}{*}{ Ingeniería Ambiental } & Bajo & $13 \%$ \\
\cline { 2 - 3 } & Medio & $80 \%$ \\
\cline { 2 - 3 } & Alto & $7 \%$ \\
\hline \multirow{2}{*}{ Ingeniería Mecatrónica } & Bajo & $13 \%$ \\
\cline { 2 - 3 } & Medio & $87 \%$ \\
\hline
\end{tabular}

Los datos obtenidos en atención selectiva (Tabla 7) muestran que el $47 \%$ de estudiantes de IA y el $12 \%$ de IM tuvieron un desempeño bajo. Esto evidencia su dificultad para seleccionar información relevante, inhibiendo la atención a unos estímulos mientras se atiende a otros, por lo que la capacidad de procesar o generar un esquema apropiado tiende a ser baja. A su vez, esto se relaciona con el estudio desarrollado por Gavotto y Castellanos (2015) donde se concluye que un gran porcentaje de estudiantes solo mantiene la atención durante un $70 \%$ de la clase. Se puede inferir que los resultados de bajo rendimiento académico (prueba académica), en los estudiantes de Ingeniería, podrían deberse a las dificultades para seleccionar de forma adecuada los datos requeridos para cumplir con una tarea determinada, ya que esta modalidad atencional involucra mayor exigencia cognitiva (Portellano y García, 2008).

Tabla 7 | Atención selectiva

\begin{tabular}{|c|c|c|}
\hline Programa & \multicolumn{2}{|c|}{ Porcentaje } \\
\hline \multirow{3}{*}{ Ingeniería Ambiental } & Bajo & $47 \%$ \\
\hline & Medio & $53 \%$ \\
\hline & Alto & $0 \%$ \\
\hline
\end{tabular}

$\boldsymbol{\epsilon} \mid 166$ 


\begin{tabular}{ccc}
\hline Programa & \multicolumn{2}{c}{ Porcentaje } \\
\hline \multirow{2}{*}{ Ingeniería Mecatrónica } & Bajo & $12 \%$ \\
\cline { 2 - 3 } & Medio & $88 \%$ \\
\cline { 2 - 3 } & Alto & $0 \%$ \\
\hline
\end{tabular}

En memoria de trabajo (Tabla 8), se observa que el $67 \%$ y $63 \%$ de los estudiantes, de IA e $\mathrm{IM}$, presentan habilidad para mantener nueva información por un breve periodo de tiempo, lo que les permite tener un procesamiento cognitivo esencial para desarrollar varias actividades a un mismo tiempo (De Doreña, Blázquez, González y Gil, 2012, citado por Portellano y García, 2008).

Tabla 8|Memoria de trabajo

\begin{tabular}{ccc}
\hline Programa & \multicolumn{2}{c}{ Porcentaje } \\
\hline \multirow{2}{*}{ Ingeniería Ambiental } & Bajo & $20 \%$ \\
\cline { 2 - 3 } & Medio & $67 \%$ \\
\cline { 2 - 3 } & Alto & $13 \%$ \\
\hline \multirow{2}{*}{ Ingeniería Mecatrónica } & Bajo & $25 \%$ \\
\cline { 2 - 3 } & Medio & $63 \%$ \\
\hline
\end{tabular}

Por otra parte, los resultados obtenidos para memoria declarativa (Tabla 9) permiten determinar que el $93 \%$ y el $88 \%$ de los estudiantes, de IA e IM respectivamente, presentaron dificultades en los procesos de adquisición, retención y recuperación de algunas representaciones, lo cual puede llegar a influir en el desempeño académico. Como lo explican Mejía y Escobar (2011), este tipo de memoria se relaciona con los procesos deductivos y de observación, dado que esta memoria es la que maneja conceptos teóricos mediante la creación de redes para su posterior recuperación. Mabbott y Bisanz (2008) plantean que las fallas en este tipo de memoria se hacen presentes cuando los estudiantes no logran evocar la secuencia lógica de un estímulo. 
De lo anterior, se puede concluir que el bajo rendimiento de los estudiantes de los programas de IA e IM recae en cierta medida en la imposibilidad de recordar aspectos teóricos de asignaturas con alto contenido matemático.

Tabla 9| Memoria declarativa

\begin{tabular}{ccc}
\hline Programa & \multicolumn{2}{c}{ Porcentaje } \\
\hline \multirow{2}{*}{ Ingeniería Ambiental } & Bajo & $93 \%$ \\
\cline { 2 - 3 } & Medio & $7 \%$ \\
\cline { 2 - 3 } Ingeniería Mecatrónica & Alto & $0 \%$ \\
\cline { 2 - 3 } & Bajo & $88 \%$ \\
\cline { 2 - 3 } & Medio & $13 \%$ \\
\hline
\end{tabular}

Con respecto a las funciones ejecutivas, en el componente de actualización, se encontró que el $33 \%$ y el $25 \%$, de los estudiantes de IA e IM respectivamente, presentan bajos niveles en la monitorización, manipulación y actualización de información, definida como la inserción y eliminación de datos en la memoria a corto plazo (Miyake, Friedman, Emerson, Witzki, Howerter, \& Wager, 2000). (Tabla 10). Esto genera dificultades tanto para la organización de la nueva información y el desarrollo de las tareas asociadas al pensamiento lógico matemático como para el manejo del conocimiento científico (Vallejos, 2012).

Tabla 10|Actualización

\begin{tabular}{ccc}
\hline Programa & \multicolumn{2}{c}{ Porcentaje } \\
\hline \multirow{2}{*}{ Ingeniería Ambiental } & Bajo & $33 \%$ \\
\cline { 2 - 3 } & Medio & $60 \%$ \\
\cline { 2 - 3 } & Alto & $7 \%$ \\
\hline \multirow{2}{*}{ Ingeniería Mecatrónica } & Bajo & $25 \%$ \\
\cline { 2 - 3 } & Medio & $75 \%$ \\
\hline
\end{tabular}


Por otro lado, también se habla de la alternancia como componente de las funciones ejecutivas. Miyake et al. (2000) la describen como la capacidad de cambiar de manera flexible entre distintas operaciones mentales o esquemas. Para el caso de esta investigación, se observó que el $53 \%$ y el $75 \%$, de los estudiantes de IA e IM respectivamente, tienen dificultades en este proceso (Tabla 11). Lo que lleva a pensar que quizás esto crea deficiencias en la planificación de los procesos y en el desarrollo de las estrategias establecidas para llevar a cabo actividades de forma eficaz.

Tabla 11 |Alternancia

\begin{tabular}{ccc}
\hline Programa & \multicolumn{2}{c}{ Porcentaje } \\
\hline \multirow{2}{*}{ Ingeniería Ambiental } & Bajo & $53 \%$ \\
\cline { 2 - 3 } & Medio & $27 \%$ \\
\cline { 2 - 3 } & Alto & $20 \%$ \\
\hline \multirow{2}{*}{ Ingeniería Mecatrónica } & Bajo & $75 \%$ \\
\cline { 2 - 3 } & Medio & $12 \%$ \\
\cline { 2 - 3 } & Alto & $13 \%$ \\
\hline
\end{tabular}

En razón a lo mencionado anteriormente, se evidencia que hay un porcentaje importante de estudiantes que presenta dificultades en varios de los procesos cognitivos, objeto de estudio de la presente investigación. Lo cual podría explicar algunos de los resultados obtenidos en su proceso académico y, además, en situaciones relacionadas con resolución de problemas de la vida cotidiana que requieren monitoreo, flexibilidad, retención de información, regulación del comportamiento e inhibición de estímulos perturbadores (Vergara, 2011). De hecho, se encontró que un $20 \%$ y $25 \%$, de estudiantes de los programas de Ingeniería Ambiental y Mecatrónica respectivamente (Tabla 12), presentan dificultades en la capacidad de ignorar la información irrelevante. Sin embargo, un $67 \%$ y $63 \%$ presentan un adecuado proceso de inhibición de los diferentes estímulos que se les presentan en su diario vivir. 
Tabla 12 | Inhibición

\begin{tabular}{ccc}
\hline Programa & \multicolumn{2}{c}{ Porcentaje } \\
\hline \multirow{3}{*}{ Ingeniería Ambiental } & Bajo & $20 \%$ \\
\cline { 2 - 3 } & Medio & $67 \%$ \\
\cline { 2 - 3 } & Alto & $13 \%$ \\
\cline { 2 - 3 } Ingeniería Mecatrónica & Bajo & $25 \%$ \\
\cline { 2 - 3 } & Medio & $63 \%$ \\
\cline { 2 - 3 } & Alto & $12 \%$ \\
\hline
\end{tabular}

Para finalizar, es necesario mencionar a González (2013) quien manifiesta que el bajo rendimiento en áreas matemáticas se debe al método de enseñanza impuesto por la sociedad, que ha otorgado un lugar primordial a la memorización, en lugar de privilegiar un modelo que refuerce las funciones ejecutivas mediante la comprensión y solución de problemas. Para el desarrollo de este tipo de actividades se requieren procesos de comprensión, manipulación y organización de la información, que aumentan la probabilidad de que se genere un aprendizaje significativo y, por ende, una mejor memoria.

Los resultados obtenidos con respecto a la función ejecutiva de actualización permiten establecer que la mayoría de los participantes sí logran analizar y trasformar con éxito la información de un problema para darle solución en un esquema lógico matemático. No obstante, a un porcentaje de ellos no se les facilita este tipo de procesos, pues, como lo mencionan Farías (2009) y Morales, Alviter, Hidalgo, García y Molinar, (2013), una mínima activación de los procesos cognitivos de orden superior (funciones ejecutivas) los lleva a un bajo rendimiento académico.

\section{Conclusiones}

Se concluye que hay un porcentaje importante de estudiantes con dificultades en varios de los procesos cognitivos, siendo relevantes los procesos de memoria declarativa y la función ejecutiva relacionada con la alternancia. 


\section{Conflicto de intereses}

Cada una de las autoras declara no tener conflictos de intereses financieros ni personales que puedan influir inapropiadamente en la publicación de esta investigación.

\section{Referencias}

Castillo, M., \& Bracamonte, E. (2011). Estudio de la relación entre el estilo de aprendizaje de estudiantes de ingeniería y su rendimiento académico en matemática. XIII Conferencia interamericana de educación en matemáticas.

Cuervo, M. T., y Quijano, M. C. (2008). Las alteraciones de la atención y su rehabilitación en trauma craneoencefálico, Pensamiento Psicológico, 4 (11), 167-182. Disponible en: http://portales.puj.edu.co/psicorevista/ components/com_joomlib/ebooks/PS11-10.pdf

Farías, M. (2009). Actitudes y autorregulación en el aprendizaje de matemáticas. Tesis doctoral. Universidad central de Venezuela. Disponible en: saber.ucv.ve/jspui/.../Tesis\%20Doctoral\%20de\%20Mariana\%20Farias.pdf .

Fernández, A., y Gutiérrez, M. (2009). Atención selectiva, ansiedad, sintomatología depresiva y rendimiento académico en adolescentes. Electronic Journal of Research in Educational Psychology, 7 (1), 49-76. Disponible en: $h$ ttp://www.redalyc.org/articulo.oa?id=293121936004

Furlan, L., Kohan Cortada, A., Piemontesi, S. E., y Heredia, D. E. (2008). Autorregulación de la atención, afrontamiento y ansiedad ante los exámenes en estudiantes universitarios. XV Jornadas de Investigación y Cuarto Encuentro de Investigadores en Psicología del Mercosur, Facultad de Psicología. Disponible en http://www.aacademica.org/000-032/306

Gavotto, O., \& Castellanos, L. (2015). La estabilidad de la atención selectiva del estudiante y las técnicas didácticas desarrolladas durante la clase. Revista Digital de Investigación Educativa Conect, 51-69. Recuperado de: https://docplayer.es/10544560-La-estabilidad-de-la-atencion-selectiva-del-estudiante-y-las-tecnicas-didacticas-desarrolladas-durante-la-clase.html

González, M. D. (2013). Funciones ejecutivas y educación. Revista Argentina de neuropsicología, 23, 11-34. Recuperado de www.revneuropsi.com.ar/images/stories/pdf/gonzalezmunozranps23.pdf 
Hernández, R., Fernández, C., y Baptista, P. (2014). Metodología de la Investigación, 6 edición. México D. F., México: Mac Graw Hill.

Mabbott, D. J., \& Bisanz, J. (2008). Computational silla, corning memory, and conceptual knowledge in older children with mathematics learning disabilities. Journal of Learning Disabilities, 41, 15-28.

Mejía, E., y Escobar, H. (2011). Caracterización de procesos cognitivos de memoria, lenguaje y pensamiento, en estudiantes con bajo y alto rendimiento académico. Revista Diversitas Perspectivas en Psicología, 8 (1), 123-138.

Miyake, A., Friedman, N., Emerson, M., Witzki, A., Howerter, A., \& Wager, T. (2000). The unity and diversity of executive functions and their contributions to complex "Frontal Lobe" tasks: a latent variable analysis. Cognitive Psychology, 41, 49-100. doi: 10.1006/cogp.1999.0734

Morales, A., Alviter, L. E., Hidalgo, C., García, R. Z., y Molinar, J. E. (2013). Relación entre estilos de aprendizaje, rendimiento académico y otras variables relevantes de estudiantes universitarios, Revista Estilos de Aprendizaje, 11 (12), 151-166. Disponible en: https://dialnet.unirioja.es/servlet/articulo?codigo=456 3629\&orden=1\&info=link

Portellano, J., y García, J. (2008). Neuropsicología de la atención, las funciones ejecutivas y la memoria. Madrid, España: Síntesis.

Sáez, E., Guisasola, J., y Garmendia, M. (2013). Implementación y resultados obtenidos en una propuesta de aprendizaje basado en problemas en el grado en Ingeniera Ambiental, Revista de Docencia Universitaria, 11(1), 85-112. doi:10.4995/redu.2013.5549.

Vallejos, M. (2012). La motivación, la actitud hacia las ciencias, la ansiedad y las estrategias metacognitivas de lectura en el rendimiento de los estudiantes universitarios: un análisis longitudinal. Tesis doctoral, Universidad Complutense de Madrid. Disponible en http://eprints.ucm.es/17020/

Vásquez, S. (2009). Rendimiento académico y patrones de aprendizaje en estudiantes de ingeniería. Ingeniería y Universidad, 13(1), 105-136.

Vergara, M. (2011). Funciones ejecutivas y desempeño académico en estudiantes de primer año de Psicología de la Corporación Universitaria Minuto de Dios, en Bello Antioquia, trabajo de grado, Universidad de San Buenaventura. Disponible en http://hdl.handle.net/10819/1219

巨 172 